\title{
Novel use of Ketotifen as a cardio-protective agent in patients undergoing anthracycline chemotherapy
}

\author{
hosny elewa ${ }^{1}$, Naser elberay ${ }^{2}$, amany elsharif ${ }^{3}$, Walaa Keshk $^{4}$, and zeinab zalat ${ }^{3}$ \\ ${ }^{1}$ Horus University \\ ${ }^{2}$ Menoufia University \\ ${ }^{3}$ Al-Azhar University \\ ${ }^{4}$ Tanta University
}

January 6, 2021

\begin{abstract}
Objective: The present study aimed to investigate the possible cardioprotective effects of ketotifen and to assess its activity as an iron-chelating agent in patients receiving anthracyclines for the treatment of breast cancer. Patients \& Methods: This was a randomized, prospective, controlled clinical trial. 111 eligible patients with breast cancer (age range, 30-60 year) were scheduled to receive anthracycline chemotherapy. The patients divided into two groups: Patients $(n=56)$ assigned to The ketotifen group received ketotifen $1 \mathrm{mg}$ three times daily for six consecutive cycles of treatment, and patients assigned to The control group ( $\mathrm{n}=$ 55 ) without ketotifen treatment. The echocardiogram for each patient was recorded two times at baseline and at the end of the study. As well, blood samples were collected from all patients. Results: The findings showed a statistically significant reduction in the mean serum levels of common cardiotoxicity accompanied biomarkers in The ketotifen group compared with The control group ( $\mathrm{P}$ [?] 0.05). The mean serum levels of total iron-binding capacity was significantly elevated in The ketotifen group (P [?] 0.001). There was a direct correlation between the mean serum levels of iron and that of lactate dehydrogenase (LDH) ( $\mathrm{r}$ $=+0.79)$. On the other hand, there were indirect correlations between mean serum levels of LDH and both the percentage of ejection fraction and the total iron-binding capacity $(\mathrm{r}=-0.69$ and -0.697 , respectively). Conclusion: Oral administration of ketotifen appears to be efficient and safe as a novel cardioprotective agent for the prevention of anthracyclines induced cardiotoxicity. Additionally, ketotifen suggested a beneficial effect in iron overload inducing diseases such as COVID-19.
\end{abstract}

\section{Hosted file}

ketotifen version.edited Round 5.pdf available at https://authorea.com/users/387711/articles/ 502666-novel-use-of-ketotifen-as-a-cardio-protective-agent-in-patients-undergoinganthracycline-chemotherapy 